\title{
Values for Strategic Games in Which Players Cooperate
}

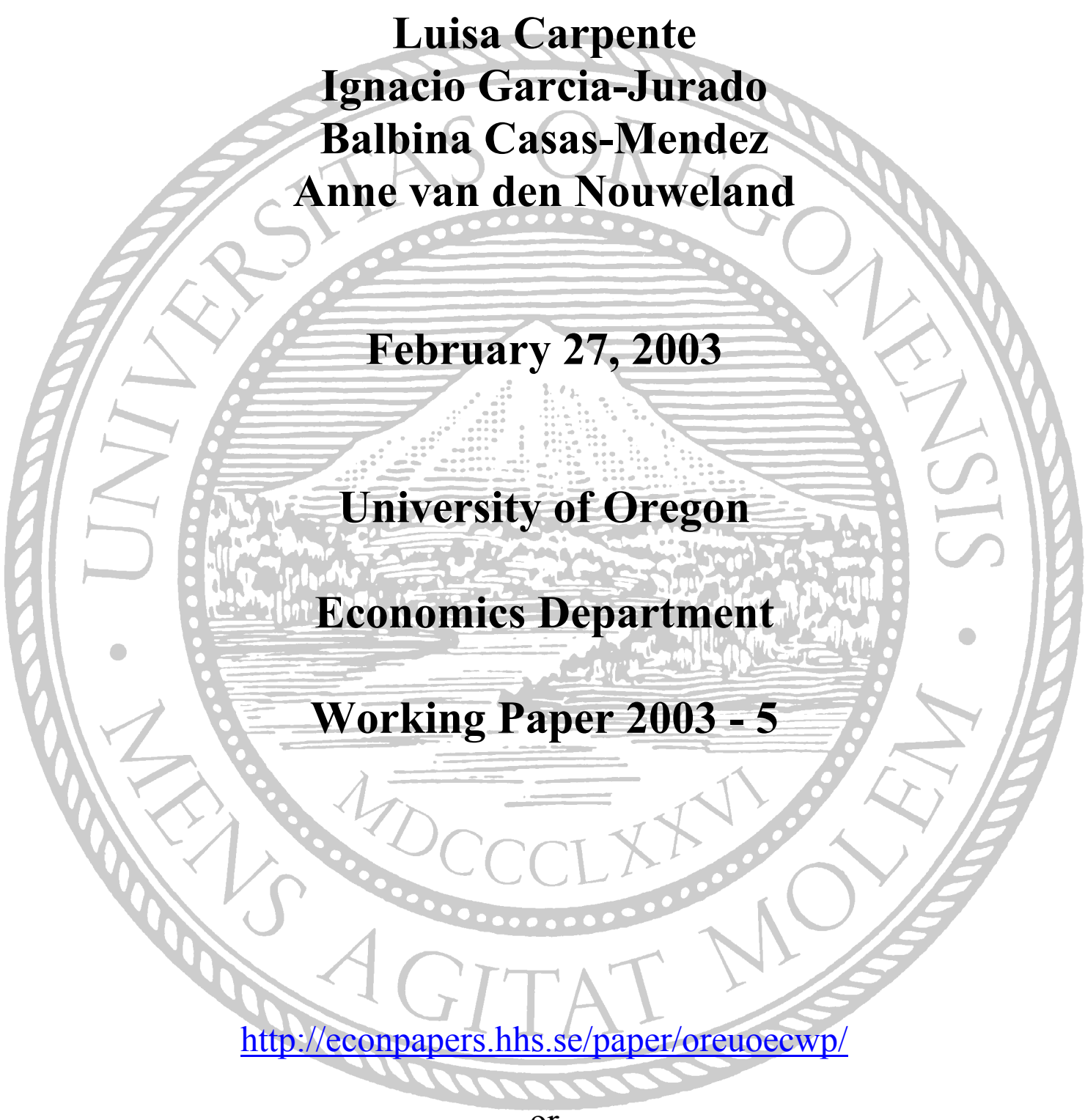

http://economics.uoregon.edu/papers 


\title{
Values for strategic games in which players cooperate* $^{*}$
}

\author{
Luisa Carpente ${ }^{1}$ \\ Balbina Casas-Méndez ${ }^{2}$ \\ Ignacio García-Jurado ${ }^{2} \quad$ Anne van den Nouweland ${ }^{3}$
}

February 27, 2003

\begin{abstract}
In this paper we propose a new method to associate a coalitional game with each strategic game. The method is based on the lower value of matrix games. We axiomatically characterize this new method, as well as the method that was described in von Neumann and Morgenstern (1944). As an intermediate step, we provide some axiomatic characterizations of the value and the lower value of matrix games.
\end{abstract}

\section{Introduction}

Classical game theory makes a radical distinction between non-cooperative games and cooperative games. Usually, non-cooperative games are defined as games that do not permit enforceable agreements among players. This

\footnotetext{
*Authors acknowledge the financial support of Spanish Ministry for Science and Technology and FEDER through projects PB98-0613-C02-02 and BEC2002-04102-C02-02.

${ }^{1}$ Departamento de Matemáticas. Facultade de Informática. Universidade da Coruña. 15071 A Coruña, Spain.

${ }^{2}$ Departamento de Estatística e IO. Facultade de Matemáticas. Universidade de Santiago de Compostela. 15782 Santiago de Compostela, Spain.

${ }^{3}$ Department of Economics, 435 PLC, University of Oregon, Eugene, OR 97403-1285, USA, and Department of Economics, The University of Melbourne, Australia. This paper was started while this author visited the University of Santiago de Compostela in the summer of 2002. Support from University of Santiago de Compostela is gratefully acknowledged.
} 
is contrast to cooperative games, in which enforceable agreements are possible. However, we think that the point of view adopted in van Damme and Furth (2002) reflects the difference between non-cooperative games and cooperative games more accurately. They write:

"The terminology that is used sometimes gives rise to confusion; it is not the case that in non-cooperative games players do not wish to cooperate and that in cooperative games players automatically do so. The difference instead is in the level of detail of the model; non-cooperative models assume that all the possibilities for cooperation have been included as formal moves in the game, while cooperative models are 'incomplete' and allow players to act outside of the detailed rules that have been specified."

This description is, in fact, more in accordance with the approach in von Neumann and Morgenstern (1944). Given a non-cooperative game, they formulate a cooperative game that describes for each coalition the benefits that this coalition can secure for its members, independently of the actions taken by the players outside the coalition. Hence, the cooperative-game description abstracts away from the details of the non-cooperative game and collapses those into simple numbers, one for each coalition of players. For a coalition of players to secure the benefits (or worth of the coalition) for its members, these members will most likely have to coordinate their actions and this in itself will generally require them to act outside the detailed rules of the non-cooperative game. To more clearly reflect the interpretations provided above, we prefer to use the terminology strategic game (instead of non-cooperative game) and coalitional game (instead of cooperative game).

The main objective of the current paper is to highlight the connection between strategic games and coalitional games. We do so by providing axiomatic foundations for two procedures that associate a coalitional game with each strategic game. The first procedure we study is that introduced in von Neumann and Morgenstern (1944), which defines the worth of a coalition of players $S$ to be the value of the mixed extension of the zero-sum game between coalition $S$ on the one hand and the coalition $N \backslash S$ consisting of all 
the other players on the other hand. In this zero-sum game, coalition $N \backslash S$ tries to keep the payoff to coalition $S$ as low as possible, while coalition $S$ tries to maximize its payoff. We also introduce a second procedure, which defines the worth of a coalition $S$ to be the lower value of the zero-sum game between coalition $S$ and coalition $N \backslash S$. The benefit of considering the lower value of the game instead of the value of its mixed extension is that the former requires no use of mixed strategies, whereas the latter does. Hence, in situations where the use of mixed strategies is not plausible, the procedure using the lower value will provide a more plausible method to determine the benefits that each coalition can secure for its members.

To provide axiomatic foundations for these two procedures we start by looking into axiomatic characterizations of the values of mixed extensions of matrix games and of the lower values of matrix games, using the characterizations of the value by Vilkas (1963) and Tijs (1981) as starting points.

We point out that there are papers that propose procedures to associate coalitional games with strategic games that are quite different from the ones we study in the current paper. Harsanyi (1963), Myerson (1991) and Bergantiños and García-Jurado (1995) are some of these papers. All of these take an approach that involves the Nash equilibrium concept rather than the value or lower value. Myerson (1978) is a paper that is remotely related to the current one. It studies the role of threats in strategic games in which players are assumed to cooperate. Finally, we remark that we do consider only situations where utilities are transferable between the players in a coalition. Procedures to associate NTU-games with strategic games were proposed in Aumann $(1961,1967)$. Aumann's work was continued in Borm and Tijs (1992).

The structure of this paper is as follows. In section 2, we provide definitions related to matrix games and we develop axiomatic characterizations of the value and the lower value of matrix games. In section 3 , we include definitions related to strategic games and coalitional games and we study methods to associate a coalitional game with each strategic game. We provide axiomatic chracterizations of the method defined in von Neumann and 
Morgenstern (1944) as well as a newly-defined method that is based on the lower value.

\section{Values of matrix games}

In this section, we concentrate on matrix games. We define and develop axiomatic characterizations of both the value of matrix games and the lower value of matrix games. These axiomatizations will be used in the next section to construct axiomatic characterizations of methods that associate coalitional games with strategic games.

A matrix game is a two-person zero-sum game $(M, N, A)$ in which $M=$ $\{1, \ldots, m\}$ and $N=\{1, \ldots, n\}$ are the strategy sets of players 1 and 2 , respectively, and $A=\left[a_{i j}\right]_{i \in M, j \in N}$ is an $m \times n$ matrix of real numbers. If player 1 chooses strategy $i \in M$ and player 2 chooses strategy $j \in N$, then player 2 pays $a_{i j}$ to player 1 , so player 1 has a payoff of $a_{i j}$ and player 2 has a payoff of $-a_{i j}$. For the sake of simplicity, we refer to the matrix game $(M, N, A)$ simply as $A$.

For a matrix game $A$, the mixed extension of $A$, denoted by $E(A)$, is a two-person zero-sum game in which the strategy set of player 1 is

$$
S_{m}=\left\{x \in \mathbb{R}^{m} \mid x_{i} \geq 0 \text { for all } i \in M, \sum_{i \in M} x_{i}=1\right\},
$$

i.e., player 1 chooses a probability distribution on his strategies, and the strategy set of player 2 is similarly defined by

$$
S_{n}=\left\{y \in \mathbb{R}^{n} \mid y_{j} \geq 0 \text { for all } j \in N, \sum_{j \in N} y_{j}=1\right\} .
$$

If player 1 chooses $x \in S_{m}$ and player 2 chooses $y \in S_{n}$, the (expected) payoff to player 1 is $x^{T} A y$ (or $\sum_{i \in M} \sum_{j \in N} x_{i} y_{j} a_{i j}$ ) and that to player 2 is $-x^{T} A y{ }^{1}$

The value of a matrix game is defined through its mixed extension using the lower and upper values. The lower and upper values of $E(A), \underline{V}(\mathrm{E}(\mathrm{A}))$

\footnotetext{
${ }^{1} x^{T}$ denotes the transpose of $x$.
} 
and $\bar{V}(E(A))$, are defined as:

$$
\begin{aligned}
& \underline{V}(E(A)):=\max _{x \in S_{m}} \min _{y \in S_{n}} x^{T} A y \\
& \bar{V}(E(A)):=\min _{y \in S_{n}} \max _{x \in S_{m}} x^{T} A y .
\end{aligned}
$$

At the basis of these definitions is that player 1 wants to maximize $x^{T} A y$ and player 2 wants to minimize it. By choosing an appropriate $x \in S_{m}$, player 1 can make sure he gets at least the lower value. Similarly, by choosing an appropriate $y \in S_{n}$, player 2 can make sure he does not have to pay more than the the upper value. Note that it follows easily from these definitions that $\underline{V}(E(A)) \leq \bar{V}(E(A)$ ). The classical minimax theorem (cf. von Neumann (1928)) asserts that for every matrix game $A$ it holds that $\underline{V}(E(A))=\bar{V}(E(A))$, so that player 1 expects to get exactly the amount $\underline{V}(E(A))$. This leads to the definition of the value of the matrix game, $V(A):=\underline{V}(E(A))=\bar{V}(E(A))$. If the players cannot use probability distributions on their strategies, we get the lower value

$$
\underline{V}(A):=\max _{i \in M} \min _{j \in N} a_{i j}
$$

of the matrix game and its upper value

$$
\bar{V}(A):=\min _{j \in N} \max _{i \in M} a_{i j} .
$$

In general, $\underline{V}(A)<\bar{V}(A)$. Note that $\underline{V}(A) \leq V(A) \leq \bar{V}(A)$ for all matrices $A$.

We denote the set of real matrices by $\mathcal{A}$. The value function $V: \mathcal{A} \rightarrow \mathbb{R}$ associates with each matrix $A \in \mathcal{A}$ its value $V(A)$. The value function is an example of a an evaluation function, which we define as a real-valued function $f: \mathcal{A} \rightarrow \mathbb{R}$ that assigns to every matrix $A \in \mathcal{A}$ a real number reflecting the evaluation of the matrix game $A$ from the point of view of player 1. The value function was characterized as an evaluation function in Vilkas (1963), whose characterization was extended to a broader class of zero-sum games in Tijs (1981). As the value function can only be applied in situations 
where mixed strategies can be used by the players, we are interested in the evaluation function based on the lower value. We axiomatically characterize this evaluation function and also provide new characterizations for the value function.

We start by recalling Vilkas's (1963) characterization of the value function. He used the following four properties of an evaluation function $f$ : $\mathcal{A} \rightarrow \mathbb{R}$.

A1 Objectivity. $f([a])=a$ for all $a \in \mathbb{R}^{2}$

A2 Monotonicity. For all $A, B \in \mathcal{A}$, if $A \geq B$, then $f(A) \geq f(B)$.

A3 Row dominance. The $i^{\text {th }}$ row of the matrix $A$, denoted

$$
r_{i}=\left[a_{i 1}, \ldots, a_{i n}\right],
$$

is dominated if there exists a convex combination $x$ of the other rows of $A^{3}$ with the property that $x_{j} \geq a_{i j}$ for all $j \in N$. For any $A \in \mathcal{A}$, if row $r$ is dominated, then $f(A)=f(A \backslash r)$, where $A \backslash r$ represents the matrix obtained from $A$ by deleting row $r$.

A4 Symmetry. $f\left(-A^{T}\right)=-f(A)$ for all $A \in \mathcal{A}$.

Objectivity establishes the evaluation for player 1 in a trivial situation where both players have exactly one strategy available. Monotonicity states that the evaluation for player 1 should not decrease when his payoff weakly increases for every possible choice of strategies by both players. Row dominance states that player 1's evaluation should not change if he can no longer choose a strategy that is worse for him than some combination of other strategies. Note that this property makes sense only in a setting where players can mix their strategies. Symmetry establishes that the roles of players 1 and 2 can be interchanged if the matrix is adapted accordingly.

\footnotetext{
${ }^{2}$ Here, $[a]$ denotes the $1 \times 1$ matrix with $a_{11}=a$.

${ }^{3} x$ is a convex combination of the other rows if there exist $\left(\alpha_{k}\right)_{k \in M \backslash i}$ such that $\alpha_{k} \geq 0$ for each $k \in M \backslash i$, with $\sum_{k \in M \backslash i} \alpha_{k}=1$, and $x=\sum_{k \in M \backslash i} \alpha_{k} r_{k}$.
} 
Transposing the matrix interchanges the roles of the players and the minus sign appears because the payoff of player 2 is the opposite of that of player 1 .

Theorem 1 (Vilkas (1963)) The value function $V$ is the unique evaluation function that satisfies objectivity (A1), monotonicity (A2), row dominance (A3), and symmetry (A4).

The counterpart for player 2 to row dominance would be what we call column dominance, which is related to dominated strategies of player two.

A5 Column dominance. The $j^{\text {th }}$ column of the matrix $A$, denoted $c_{j}=\left[a_{1 j}, \ldots, a_{m j}\right]$, is dominated if there exists a convex combination $y$ of the other columns of $A$ with the property that $y_{i} \leq a_{i j}$ for all $i \in M$. For any $A \in \mathcal{A}$, if column $c$ is dominated, then $f(A)=f(A \backslash c)$, where $A \backslash c$ represents the matrix obtained from $A$ by deleting column $c$.

Column dominance states that player 1's evaluation should not change if player 2 can no longer choose a strategy that is dominated for him by some combination of his other strategies. Note that player 2 wants to minimize the payoff of player 1 (thereby maximizing his own payoff), so a strategy for player 2 is dominated if it always gives a (weakly) larger payoff to player 1. Column dominance is the flip side of row dominance and can in fact replace symmetry in the characterization of the value function.

Theorem 2 The value function $V$ is the unique evaluation function that satisfies objectivity (A1), monotonicity (A2), row dominance (A3), and column dominance (A5).

Proof. It follows from Theorem 1 that $V$ satisfies $\mathrm{A} 1, \mathrm{~A} 2$, and $\mathrm{A} 3$. To see that $V$ satisfies A5, note that if $c$ is a dominated column in $A$, then this column becomes a dominated row in the matrix $-A^{T}$ and can be eliminated by A3. Symmetry assures that we can switch from the matrix to the negative of its transpose and back after the elimination of the dominated row to obtain the matrix $A \backslash c$. 
To show unicity, let $f: \mathcal{A} \rightarrow \mathbb{R}$ be an evaluation function satisfying A1, $\mathrm{A} 2, \mathrm{~A} 3$, and $\mathrm{A} 5$ and fix a matrix $A=\left[a_{i j}\right]_{i \in M, j \in N} \in \mathcal{A}$. Because $V(A)=$ $\underline{V}(E(A))$, there exists a mixed strategy of player 1 that guarantees player 1 a payoff of at least $V(A)$. Then, using A3, we can add a dominated row in which all elements equal $V(A)$ to the matrix without changing the value. Similarly, using that $V(A)=\bar{V}(E(A))$ and $\mathrm{A} 5$, we can add a dominated column in which all elements equal $V(A)$ without changing the value. Hence,

$$
f(A)=f\left(\left[\begin{array}{ccc}
a_{11} & \cdots & a_{1 n} \\
\vdots & \ddots & \vdots \\
a_{m 1} & \cdots & a_{m n} \\
V(A) & \cdots & V(A)
\end{array}\right]\right)=f\left(\left[\begin{array}{cccc}
a_{11} & \cdots & a_{1 n} & V(A) \\
\vdots & \ddots & \vdots & \vdots \\
a_{m 1} & \cdots & a_{m n} & V(A) \\
V(A) & \cdots & V(A) & V(A)
\end{array}\right]\right)
$$

Now, we use A2 to make all the elements of the matrix less than or equal to $V(A)$. This makes all the rows but the last one dominated. Hence, we can use A3 (repeatedly) to one by one eliminate all rows but the last one. Then we have a $1 \times n+1$ matrix left in which all elements equal $V(A)$. We subsequently use A5 (repeatedly) to eliminate all but one of the columns of the remaining matrix. This leaves us with a $1 \times 1$ matrix, to which we can apply A1. Doing so, we obtain

$$
\begin{gathered}
f\left(\left[\begin{array}{cccc}
a_{11} & \cdots & a_{1 n} & V(A) \\
\vdots & \ddots & \vdots & \vdots \\
a_{m 1} & \cdots & a_{m n} & V(A) \\
V(A) & \cdots & V(A) & V(A)
\end{array}\right]\right) \geq \\
f\left(\left[\begin{array}{llll}
\min \left\{a_{11}, V(A)\right\} & \cdots & \min \left\{a_{1 n}, V(A)\right\} & V(A) \\
\vdots & \ddots & \vdots & \vdots \\
\min \left\{a_{m 1}, V(A)\right\} & \cdots & \min \left\{a_{m n}, V(A)\right\} & V(A) \\
V(A) & \cdots & V(A) & V(A)
\end{array}\right]\right)= \\
f([V(A), \ldots, V(A)])=f([V(A)])=V(A) .
\end{gathered}
$$

Making all the elements of the matrix more than or equal to $V(A)$, and using A2, A5, A3, and A1, respectively, we derive in a similar manner that 


$$
\begin{gathered}
f\left(\left[\begin{array}{cccc}
a_{11} & \cdots & a_{1 n} & V(A) \\
\vdots & \ddots & \vdots & \vdots \\
a_{m 1} & \cdots & a_{m n} & V(A) \\
V(A) & \cdots & V(A) & V(A)
\end{array}\right]\right) \leq \\
f\left(\left[\begin{array}{llll}
\max \left\{a_{11}, V(A)\right\} & \cdots & \max \left\{a_{1 n}, V(A)\right\} & V(A) \\
\vdots & \ddots & \vdots & \vdots \\
\max \left\{a_{m 1}, V(A)\right\} & \cdots & \max \left\{a_{m n}, V(A)\right\} & V(A) \\
V(A) & \cdots & V(A) & V(A)
\end{array}\right]\right)= \\
f\left(\left[\begin{array}{c}
V(A) \\
\vdots \\
V(A)
\end{array}\right]\right)=f([V(A)])=V(A) .
\end{gathered}
$$

Putting together all the (in)equalities that we have derived, we obtain that $f(A)=V(A)$. This shows the value function $V$ is the only evaluation function that satisfies A1, A2, A3, and A5.

Theorems 1 and 2 show that symmetry and column dominance are equivalent in the presence of objectivity, monotonicity, and row dominance. However, this equivalence does not hold in general. For example, the evaluation function defined by $f(A)=a_{11}$, for all $A \in \mathcal{A}$, satisfies symmetry (as well as objectivity and monotonicity) but does not satisfy column dominance (or row dominance).

Row dominance and column dominance state that the elimination of a dominated strategy of player 1 or 2 has no effect on the evaluation of player 1. A natural question at this point is what the effect is of the elimination of an arbitrary strategy, dominated or not. The following two properties deal with this question.

A6 Row elimination. $f(A) \geq f(A \backslash r)$ for any row $r$ of $A$ and all $A \in \mathcal{A}$.

A7 Column elimination. $f(A) \leq f(A \backslash c)$ for any column $c$ of $A$ and all $A \in \mathcal{A}$. 
Row elimination states that player 1's evaluation should not increase when a strategy of player 1 is eliminated. Basically, it means that player 1 cannot be better off when his possibilities are further restricted. Column elimination states that the same is true for player 2; as player 2's payoff is the opposite of that of player 1, player 1's evaluation should not decrease when a strategy of player 2 is eliminated. It is not hard to see that the value function satisfies both row elimination and column elimination. These two properties highlight that the value function satisfies a form of monotonicity with respect to the elimination of strategies. The question arises whether monotonicity could be replaced by row elimination and column elimination in the previous results. The answer is affirmative.

Theorem 3 The value function $V$ is the unique evaluation function that satisfies objectivity (A1), row dominance (A3), column dominance (A5), row elimination (A6), and column elimination (A7).

Proof. We already established that $V$ satisfies A1, A3, and A5. To see that it also satisfies A6 and A7, it suffices to note that taking the maximum over a smaller set leads to a weakly smaller value and that taking the minimum over a smaller set leads to a weakly larger value.

The proof of unicity is analogue to that in Theorem 2. Let $f: \mathcal{A} \rightarrow \mathbb{R}$ be an evaluation function that satisfies the five properties and let $A=$ $\left[a_{i j}\right]_{i \in M, j \in N}$. Then, by A3 and A5 we have

$$
f(A)=f\left(\left[\begin{array}{ccc}
a_{11} & \cdots & a_{1 n} \\
\vdots & \ddots & \vdots \\
a_{m 1} & \cdots & a_{m n} \\
V(A) & \cdots & V(A)
\end{array}\right]\right)=f\left(\left[\begin{array}{cccc}
a_{11} & \cdots & a_{1 n} & V(A) \\
\vdots & \ddots & \vdots & \vdots \\
a_{m 1} & \cdots & a_{m n} & V(A) \\
V(A) & \cdots & V(A) & V(A)
\end{array}\right]\right) .
$$

Using A6, A5 and A1, respectively, we obtain

$$
f\left(\left[\begin{array}{cccc}
a_{11} & \cdots & a_{1 n} & V(A) \\
\vdots & \ddots & \vdots & \vdots \\
a_{m 1} & \cdots & a_{m n} & V(A) \\
V(A) & \cdots & V(A) & V(A)
\end{array}\right]\right) \geq f([V(A), \ldots, V(A)])
$$




$$
=f([V(A)])=V(A) .
$$

In a similar way, using $\mathrm{A} 7, \mathrm{~A} 3$ and $\mathrm{A} 1$, respectively, we have

$$
f\left(\left[\begin{array}{cccc}
a_{11} & \cdots & a_{1 n} & V(A) \\
\vdots & \ddots & \vdots & \vdots \\
a_{m 1} & \cdots & a_{m n} & V(A) \\
V(A) & \cdots & V(A) & V(A)
\end{array}\right]\right) \leq f\left(\left[\begin{array}{c}
V(A) \\
\vdots \\
V(A)
\end{array}\right]\right)=f([V(A)])=V(A)
$$

We conclude that $f(A)=V(A)$.

Monotonicity is a very different property from column elimination and row elimination, even though it can be replaced by these two properties in characterizing the value function. Monotonicity deals with a matrix of the same dimensions in which the elements change, whereas row and column elimination deal with matrices of changing dimension. The same example as we used before, the evaluation function $f$ with $f(A)=a_{11}$ for all $A \in$ $\mathcal{A}$, satisfies monotonicity but does not satisfy column elimination or row elimination.

The remainder of this section is devoted to the lower value function $\underline{V}: \mathcal{A} \rightarrow \mathbb{R}$, which assigns to every matrix $A \in \mathcal{A}$ its lower value $\underline{V}(A)$. The main advantage of the lower value function is that it does not assume that the players have preferences over lotteries; it only considers pure strategies of the matrix game. Because of this reason, this evaluation function is more appropriate to apply to wider classes of two-person zero-sum games.

To try and better understand the lower value function, we first ask ourselves which ones of the axioms used by Vilkas (1963) are satisfied by this evaluation function. Obviously, the lower value function does satisfy objectivity and monotonicity. However, the following two examples show that it does not satisfy row dominance or symmetry.

Example 1 Consider the two matrices 


$$
A=\left(\begin{array}{ll}
5 & 0 \\
1 & 4
\end{array}\right) \quad \text { and } \quad A^{\prime}=\left(\begin{array}{ll}
5 & 0 \\
1 & 4 \\
3 & 2
\end{array}\right)
$$

Note that $A^{\prime}$ is obtained from $A$ by adding a row that is a convex combination of the other rows, namely $\frac{1}{2}$ times the first row plus $\frac{1}{2}$ times the second one. To find the lower value of $A$, note that player 1 gets at least 0 if he chooses the first row, whereas he gets at least 1 if he chooses the second row. Hence, $\underline{V}(A)=1$. Similarly, we derive that $\underline{V}\left(A^{\prime}\right)=2$. Hence, the elimination of the dominated third row from $A^{\prime}$ changes the lower value and this is a violation of row dominance.

Example 2 Consider the matrices $A$ and $-A^{T}$ below.

$$
A=\left(\begin{array}{ll}
2 & 0 \\
1 & 2
\end{array}\right) \quad-A^{T}=\left(\begin{array}{ll}
-2 & -1 \\
0 & -2
\end{array}\right)
$$

It holds that $\underline{V}(A)=1$ and $\underline{V}\left(-A^{T}\right)=-2$, which illustrates that $\underline{V}$ does not satisfy symmetry. ${ }^{4}$

The lower value does satisfy the following property, which is a weaker form of row dominance.

A8 Weak row dominance. The $i^{\text {th }}$ row of the matrix $A$, denoted $r_{i}=$ $\left[a_{i 1}, \ldots, a_{i n}\right]$, is strongly dominated if there exists a row $r_{k}(k \neq i)$ in the matrix that is weakly larger than row $r_{i}$, i.e., $a_{k j} \geq a_{i j}$ for all $j \in N$. For any $A \in \mathcal{A}$, if row $r$ is strongly dominated, then $f(A)=f(A \backslash r)$.

Note that every row that is strongly dominated is also dominated but that the reverse is not necessarily true. Therefore, every row that can be eliminated under weak row dominance can also be eliminated under row dominance but not the other way around. Hence, weak row dominance is a weaker property than row dominance.

\footnotetext{
${ }^{4}$ Note that $V(A)=\frac{4}{3}$, which is obtained by player 1 playing the first row with probability $\frac{1}{3}$ and the second row with probability $\frac{2}{3}$. Also, $V\left(-A^{T}\right)=-\frac{4}{3}$, which is obtained by player 1 playing the first row of $-A^{T}$ with probability $\frac{2}{3}$ and its second row with probability $\frac{1}{3}$.
} 
In addition to objectivity, monotonicity, and weak row dominance, the lower value function satisfies column dominance. However, taking into account Theorem 2, it is clear that these four properties cannot characterize the lower value function. We need a property that is satisfied by the lower value function but not by the value function. Strong colunm dominance is such a property.

A9 Strong column dominance. The $j^{\text {th }}$ column of the matrix $A$, denoted $c_{j}=\left[a_{1 j}, \ldots, a_{m j}\right]$, is weakly dominated if for every $i \in M$ there exists an other column $c_{k}(k \neq j)$ such that $a_{i k} \leq a_{i j}$. For any $A \in \mathcal{A}$, if column $c$ is weakly dominated, then $f(A)=f(A \backslash c)$.

Weak domination of a column is a fairly weak requirement. It means that for every row, there is another column which has a weakly lower value in that row than the weakly dominated column does. Note that this can be a different column for every row. Because a column that is dominated is also weakly dominated, strong column dominance is a stronger property than column dominance. This stronger property is satisfied by the lower value function, which in fact can be characterized using this property. It is not satisifed by the value function. ${ }^{5}$

Theorem 4 The lower value function $\underline{V}$ is the unique evaluation function that satisfies objectivity (A1), monotonicity (A2), weak row dominance (A8), and strong column dominance (A9).

Proof. It is easily seen that $\underline{V}$ satisfies $\mathrm{A} 1$ and A2. To see that $\underline{V}$ satisfies A8, note that if row $r_{i}$ is strongly dominated by row $r_{k}$ in matrix $A$, then $\min _{j \in N} a_{i j} \leq \min _{j \in N} a_{k j}$. Hence, player 1 does not need row $r_{i}$ to reach the maximum of these expressions, which equals $\underline{V}(A)$. To see that $\underline{V}$ satisfies A9, note that if column $c_{j}$ is weakly dominated in matrix $A$, then

\footnotetext{
${ }^{5}$ To see this directly, consider, for example, the matrix $A=\left(\begin{array}{lll}3 & 0 & 1 \\ 0 & 3 & 1\end{array}\right)$, in which the third column is weakly dominated. However, the deletion of this column changes the value from 1 to $1 \frac{1}{2}$.
} 
$\min _{k \in N} a_{i k}=\min _{k \in N \backslash j} a_{i k}$ for every $i \in M$. Hence, deleting this column does not change the lower value.

To prove that there is no other evaluation function that satisfies $\mathrm{A} 1$, $\mathrm{A} 2, \mathrm{~A} 8$, and $\mathrm{A} 9$, let $f: \mathcal{A} \rightarrow \mathbb{R}$ be an evaluation function satisfying these properties and take a matrix $A=\left[a_{i j}\right]_{i \in M, j \in N} \in \mathcal{A}$. Suppose, without loss of generality, that $\underline{V}(A)$ is the element in the $i^{\text {th }}$ row and the $j^{\text {th }}$ column. First, we use A2 to make all the rows strongly dominated by the $i^{\text {th }}$ row. Then we apply A8 (repeatedly) to delete all the other rows. We are then left with a $1 \times n$ matrix consisting of the $i^{\text {th }}$ row of $A$. Because $\underline{V}(A)=a_{i j}$, we know that $a_{i k} \geq a_{i j}$ for all $k \in N$. Hence, in the $1 \times n$ matrix that we have left, all columns different from the $j^{\text {th }}$ are weakly dominated and can be eliminated by A9. Then we can apply A1. Doing this, we obtain

$$
\begin{gathered}
f(A) \geq f\left(\left[\begin{array}{ccc}
\min \left\{a_{11}, a_{i 1}\right\} & \cdots & \min \left\{a_{1 n}, a_{i n}\right\} \\
\vdots & \ddots & \vdots \\
\min \left\{a_{m 1}, a_{i 1}\right\} & \cdots & \min \left\{a_{m n}, a_{i n}\right\}
\end{array}\right]\right)= \\
f\left(\left[a_{i 1}, \ldots, a_{i n}\right]\right)=f\left(\left[a_{i j}\right]\right)=a_{i j}=\underline{V}(A) .
\end{gathered}
$$

To show that $f(A) \leq \underline{V}(A)$, we first add a column to the matrix $A$ in which all elements are equal to $\underline{V}(A)$. Note that such a column is weakly dominated, so by A9, this addition will not alter the lower value. Then we apply A2 to make all columns weakly dominated by the newly added one, after which we use A9 again (repeatedly) to eliminate all these other columns. We are then left with a $m \times 1$ matrix in which all elements are equal to $\underline{V}(A)$, in which all rows are strongly dominated so that we can eliminate all but one of them by A8. Application of A1 finishes the following sequence.

$$
\begin{gathered}
f(A)=f\left(\left[\begin{array}{cccc}
a_{11} & \cdots & a_{1 n} & \underline{V}(A) \\
\vdots & \ddots & \vdots & \vdots \\
a_{m 1} & \cdots & a_{m n} & \underline{V}(A)
\end{array}\right]\right) \leq \\
f\left(\left[\begin{array}{cccc}
\max \left\{a_{11}, \underline{V}(A)\right\} & \cdots & \max \left\{a_{1 n}, \underline{V}(A)\right\} & \underline{V}(A) \\
\vdots & \ddots & & \vdots \\
\max \left\{a_{m 1}, \underline{V}(A)\right\} & \cdots & \max \left\{a_{m n}, \underline{V}(A)\right\} & \underline{V}(A)
\end{array}\right]\right)=
\end{gathered}
$$




$$
f\left(\left[\begin{array}{c}
\underline{V}(A) \\
\vdots \\
\underline{V}(A)
\end{array}\right]\right)=f([\underline{V}(A)])=\underline{V}(A) .
$$

We have shown that $f(A)=\underline{V}(A)$, which proves that the lower value $\underline{V}$ is the unique evaluation function that satisfies A1, A2, A8, and A9.

Like in the characterization of the value function, monotonicity can be substituted by column elimination and row elimination in Theorem 4 .

Theorem 5 The lower value function $\underline{V}$ is the unique evaluation function that satisfies objectivity (A1), row elimination (A6), column elimination (A7), weak row dominance (A8), and strong column dominance (A9).

Proof. We already established that $V$ satisfies A1, A8, and A9. To see that it also satisfies A6 and A7, it suffices to note that taking the maximum over a smaller set leads to a weakly smaller value and that taking the minimum over a smaller set leads to a weakly larger value.

The proof of unicity is analogue to that in Theorem 4 . Let $f: \mathcal{A} \rightarrow \mathbb{R}$ be an evaluation function that satisfies A1, A6, A7, A8, and A9 and let $A=\left[a_{i j}\right]_{i \in M, j \in N} \in \mathcal{A}$. Suppose, without loss of generality, that $\underline{V}(A)$ is the element in the $i^{\text {th }}$ row and the $j^{\text {th }}$ column. Then, applying A6, A9 and A1, respectively, we obtain

$$
f(A) \geq f\left(\left[a_{i 1}, \ldots, a_{i n}\right]\right)=f\left(\left[a_{i j}\right]\right)=a_{i j}=\underline{V}(A) .
$$

Using A9, A7, A8, and A1, we obtain

$$
\begin{gathered}
f(A)=f\left(\left[\begin{array}{cccc}
a_{11} & \cdots & a_{1 n} & \underline{V}(A) \\
\vdots & \ddots & \vdots & \vdots \\
a_{m 1} & \cdots & a_{m n} & \underline{V}(A)
\end{array}\right]\right) \leq f\left(\left[\begin{array}{c}
\underline{V}(A) \\
\vdots \\
\underline{V}(A)
\end{array}\right]\right)= \\
f([\underline{V}(A)])=\underline{V}(A) .
\end{gathered}
$$


This proves that $f(A)=\underline{V}(A)$.

We conclude this section with some comments on two properties that we did not encounter in the characterization results above, but that naturally come to mind.

A10 Weak column dominance. The $j^{\text {th }}$ column of the matrix $A$, denoted $c_{j}=\left[a_{1 j}, \ldots, a_{m j}\right]$, is strongly dominated if there exists a column $c_{k}$ $(k \neq j)$ in the matrix that is weakly larger than column $c_{j}$, i.e., $a_{i k} \leq$ $a_{i j}$ for all $i \in M$. For any $A \in \mathcal{A}$, if column $c$ is strongly dominated, then $f(A)=f(A \backslash c)$.

A11 Strong row dominance. The $i^{\text {th }}$ row of the matrix $A$, denoted $r_{i}=\left[a_{i 1}, \ldots, a_{i n}\right]$, is weakly dominated if for every $j \in N$ there exists an other row $r_{k}(k \neq i)$ such that $a_{i j} \leq a_{i k}$. For any $A \in \mathcal{A}$, if row $r$ is weakly dominated, then $f(A)=f(A \backslash r)$.

These properties are the counterparts to weak row dominance and strong column dominance. Column dominance implies weak column dominance and strong row dominance implies row dominance. Hence, both the value function and the lower value function satisfy weak column dominance because they both satisfy column dominance. The lower value function does not satisfy strong row dominance because it does not satisfy row dominance. The value function does not satisfy strong row dominance either, as can be seen by considering the matrix $A=\left(\begin{array}{ll}3 & 0 \\ 0 & 3 \\ 2 & 2\end{array}\right)$, in which the third row is weakly dominated. However, the deletion of this row changes the value from 2 to $1 \frac{1}{2}$.

In the following table we provide an overview of the various properties that we have encountered in this section and for each property we indicate whether it is satisfied $(\checkmark)$ by the value function and the lower value function or not $(-)$. 


\begin{tabular}{|l|c|c|}
\hline & $V$ & $\underline{V}$ \\
\hline Objectivity & $\checkmark$ & $\checkmark$ \\
\hline Monotonicity & $\checkmark$ & $\checkmark$ \\
\hline Symmetry & $\checkmark$ & - \\
\hline Row elimination & $\checkmark$ & $\checkmark$ \\
\hline Column elimination & $\checkmark$ & $\checkmark$ \\
\hline Weak row dominance & $\checkmark$ & $\checkmark$ \\
\hline Row dominance & $\checkmark$ & - \\
\hline Strong row dominance & - & - \\
\hline Weak column dominance & $\checkmark$ & $\checkmark$ \\
\hline Column dominance & $\checkmark$ & $\checkmark$ \\
\hline Strong column dominance & - & $\checkmark$ \\
\hline
\end{tabular}

\section{Characteristic functions associated with strate- gic games.}

In this section, we consider methods to associate a characteristic function with each strategic game. We consider the method defined in von Neumann and Morgenstern (1944), which is based on the value function, and we also introduce a new method based on the lower value function. We provide axiomatic foundations for both methods, based on our axiomatizations of the value function and the lower value function. We remind the reader that the motivation for considering methods to associate a characteristic function with each strategic game is to tackle those situations in which players can act outside the rules of the strategic game, which only describes the actions available to each of the players and their payoff functions, but does not detail all their cooperative possibilities. We start by providing the necessary definitions.

A strategic game $g=\left(N,\left\{X_{i}\right\}_{i \in N},\left\{u_{i}\right\}_{i \in N}\right)$ consists of a set of players $N=\{1, \ldots, n\}$, and for every player $i \in N$ a set of strategies $X_{i}$ available to this player, and a payoff function $u_{i}: \prod_{j \in N} X_{j} \rightarrow \mathbb{R}$. In this paper we consider only finite strategic games ${ }^{6}$, which are those games in which the

\footnotetext{
${ }^{6}$ However, some results we obtain can easily be extended to wider classes of strategic games.
} 
strategy sets $\left\{X_{i}\right\}_{i \in N}$ are all finite. The class of finite strategic games with player set $N$ is denoted by $G^{N}$. We denote the class of all finite strategic games by $G$.

A coalitional game is a pair $(N, v)$, where $N=\{1, \ldots, n\}$ is the set of players and $v: 2^{N} \rightarrow \mathbb{R}$ is the characteristic function of the game, assigning to each coalition $S \subset N$ its worth $v(S)$. The worth $v(S)$ of a coalition $S$ represents the benefits that this coalition can guarantee its members independently of what the other players (those in $N \backslash S$ ) do. By convention, $v(\emptyset)=0$. From now on, we identify a coalitional game $(N, v)$ with its characteristic function $v$. We denote the class of coalitional games with player set $N$ by $\Gamma^{N}$ and we use $\Gamma$ to denote the class of all coalitional games. A coalitional game $v \in \Gamma^{N}$ is said to be superadditive if $v(S \cup T) \geq v(S)+v(T)$ for all coalitions $S, T \subset N$ with $S \cap T=\emptyset$.

Von Neumann and Morgenstern (1944) propose the following procedure to associate a coalitional game with every strategic game. Let $g \in G^{N}$ be a strategic game and take a non-empty coalition $S \subset N, S \neq N$. The 2-person zero-sum game $g_{S}$ is defined by

$$
g_{S}=\left(\{S, N \backslash S\},\left\{X_{S}, X_{N \backslash S}\right\},\left\{u_{S},-u_{S}\right\}\right),
$$

where, for all $T \subset N, X_{T}=\prod_{i \in T} X_{i}$ and $u_{T}=\sum_{i \in T} u_{i}$. In this game, there are two players, coalition $S$ and coalition $N \backslash S$. The strategies available to each of these two coalitions are all the combinations of the strategies available to its members in the game $g$. The payoff to coalition $S$ is the sum of the payoffs of its members for every possible strategy tuple, and the payoff to coalition $N \backslash S$ is the opposite of this. Note that the game $g_{S}$ is essentially a matrix game. We denote by $A_{S}$ the matrix of this game. Now, in the coalitional game $v_{g} \in \Gamma^{N}$ associated with the strategic game $g$, the worth of coalition $S$ is the value of this matrix game;

$$
v_{g}(S)=V\left(A_{S}\right)
$$

This is the worth that the coalition $S$ can secure for itself even if the players in $N \backslash S$ cooperate to keep the worth of coalition $S$ as low as possible. The 
worth of the grand coalition $N$ is simply defined as $v_{g}(N)=\max _{x \in X_{N}} u_{N}(x)$. The interpretation of $v_{g}$ is that the players in a coalition $S$ assume that all the players who are not in the coalition will coordinate their strategy choices to try and keep the payoff to $S$ as small as possible. Note that the value of $A_{S}$ is defined using the mixed extension of the matrix game $A_{S}$, so that it is implicitly assumed that the players in a coalition cannot only coordinate their pure strategies, but can even choose a probability distribution over their coordinated strategies. This is a very strong assumption.

The philosophy underlying von Neumann and Morgenstern's procedure is intimately connected to the characteristic function concept. Since the characteristic function provides the benefits that every coalition can guarantee its members, independently of what the other players do, von Neumann and Morgenstern's procedure seems to be a sensible one, at least in situations in which coalitions of players have preferences over lotteries and in which their utility functions are linear.

However, in settings in which mixing coordinated strategies is not possible or reasonable, it is more appropriate to stick to pure strategies and the lower value of the matrix game. This leads us to associate with a strategic game $g \in G^{N}$ the coalitional game $w_{g} \in \Gamma^{N}$ defined by

$$
w_{g}(S)=\underline{V}\left(A_{S}\right)
$$

for all non-empty $S \subset N, S \neq N$, and $w_{g}(N)=\max _{x \in X_{N}} u_{N}(x)$. Note that this coalitional game is more pessimistic than von Neumann and Morgenstern's, in the sense that $w_{g}(S) \leq v_{g}(S)$ for all $g \in G^{N}$ and all $S \subset N .^{7}$ We illustrate both games in the following example.

Example 3 Consider the following three-player strategic game g, in which player 1 is the row player, player 2 the column player, and player 3 chooses the matrix to the left or that to the right.

\begin{tabular}{|c|c|c|}
\hline$\alpha_{3}$ & $\alpha_{2}$ & $\beta_{2}$ \\
\hline$\alpha_{1}$ & $(1,1,1)$ & $(0,0,0)$ \\
\hline$\beta_{1}$ & $(0,0,0)$ & $(1,1,1)$ \\
\hline
\end{tabular}

\begin{tabular}{|c|c|c|}
\hline$\beta_{3}$ & $\alpha_{2}$ & $\beta_{2}$ \\
\hline$\alpha_{1}$ & $(0,0,0)$ & $(1,1,1)$ \\
\hline$\beta_{1}$ & $(1,1,1)$ & $(0,0,0)$ \\
\hline
\end{tabular}

\footnotetext{
${ }^{7}$ This follows from $\underline{V}(A) \leq V(A)$ for all matrices $A$.
} 
First, consider the coalition $S=\{1\}$. The matrix of the 2-person zerosum game associated with this coalition is $A_{S}=\left(\begin{array}{llll}1 & 0 & 0 & 1 \\ 0 & 1 & 1 & 0\end{array}\right)$, where the rows are ordered as before and the columns are ordered as follows. The first column corresponds to the strategies $\left(\alpha_{2}, \alpha_{3}\right)$ by $N \backslash S=\{2,3\}$, the second column to $\left(\beta_{2}, \alpha_{3}\right)$, the third column to $\left(\alpha_{2}, \beta_{3}\right)$, and the fourth column to $\left(\beta_{2}, \beta_{3}\right)$. The value of this matrix is $V\left(A_{S}\right)=\frac{1}{2}$ and its lower value equals $\underline{V}\left(A_{S}\right)=0$. Hence, we find that $v_{g}(1)=\frac{1}{2}$ and $w_{g}(1)=0 .{ }^{8}$ Applying the same procedure to the coalitions $\{2\}$ and $\{3\}$, we find $v_{g}(2)=v_{g}(3)=\frac{1}{2}$ and $w_{g}(2)=w_{g}(3)=0$.

Now, let us consider a 2-player coalition, say $S=\{1,2\}$. The matrix of the 2-person zero-sum game associated with this coalition is $A_{S}=$ $\left(\begin{array}{ll}2 & 0 \\ 0 & 2 \\ 0 & 2 \\ 2 & 0\end{array}\right)$, where the columns correspond to the strategies $\alpha_{3}$ and $\beta_{3}$ (from left to right) of player $3 \in N \backslash S$ and the rows are ordered as follows. The first row corresponds to the strategies $\left(\alpha_{1}, \alpha_{2}\right)$ by the players in $S$, the second row to $\left(\beta_{1}, \alpha_{2}\right)$, the third row to $\left(\alpha_{1}, \beta_{2}\right)$, and the fourth row to $\left(\beta_{1}, \beta_{2}\right)$. The value of this matrix is $V\left(A_{S}\right)=1$ and its lower value equals $\underline{V}\left(A_{S}\right)=0$. Hence, we find that $v_{g}(1,2)=1$ and $w_{g}(1,2)=0$. Applying the same procedure to the coalitions $\{1,3\}$ and $\{2,3\}$, we find $v_{g}(1,3)=v_{g}(2,3)=1$ and $w_{g}(1,3)=w_{g}(2,3)=0$.

For the grand coalition, we find $v_{g}(N)=w_{g}(N)=3$.

This example illustrates that in general the two coalitional games $v_{g}$ and $w_{g}$ are different and also that $w_{g}(S) \leq v_{g}(S)$ for all coalitions $S \subset N$.

Note that the games $v_{g}$ and $w_{g}$ that we derived in the previous example are both superadditive. This is not a coincidence, but holds for all coalitional games derived in the described manner from strategic games using the value or the lower value. For the games $v_{g}$ this was shown in von Neumann and Morgenstern (1944) and for the games $w_{g}$ we show this in the following

\footnotetext{
${ }^{8}$ Note that we omit the set-brackets $\{$ and $\}$ around 1 . This is often done in the literature and we adopt this convention.
} 
Proposition.

Proposition 1 For every strategic game $g \in G^{N}$ it holds that the associated coalitional game $w_{g}$ is superadditive.

Proof. Let $g=\left(N,\left\{X_{i}\right\}_{i \in N},\left\{u_{i}\right\}_{i \in N}\right) \in G^{N}$ be a strategic game and take two non-empty coalitions $S, T \subset N$, such that $S \cap T=\emptyset$. Then

$$
\begin{gathered}
w_{g}(S \cup T)=\max _{x_{S T} \in X_{S \cup T}} \min _{-S T} \in X_{N \backslash(S \cup T)} u_{S \cup T}\left(x_{S T}, x_{-S T}\right) \\
=\max _{x_{S} \in X_{S}} \max _{x_{T} \in X_{T}} \min _{x_{-S T} \in X_{N \backslash(S \cup T)}} u_{S \cup T}\left(x_{S}, x_{T}, x_{-S T}\right) .
\end{gathered}
$$

Now, fix a $y_{S} \in X_{S}$ and a $y_{T} \in X_{T}$. We can now derive that

$$
\begin{gathered}
w_{g}(S \cup T) \geq \min _{x_{-S T} \in X_{N \backslash(S \cup T)}} u_{S \cup T}\left(y_{S}, y_{T}, x_{-S T}\right) \\
\geq \min _{x_{-S T} \in X_{N \backslash(S \cup T)}} u_{S}\left(y_{S}, y_{T}, x_{-S T}\right)+\min _{x_{-S T} \in X_{N \backslash(S \cup T)}} u_{T}\left(y_{S}, y_{T}, x_{-S T}\right) \\
\quad \geq \min _{x_{T} \in X_{T}} \min _{x_{-S T} \in X_{N \backslash(S \cup T)}} u_{S}\left(y_{S}, x_{T}, x_{-S T}\right) \\
\quad+\min _{x_{S} \in X_{S}} \min _{x_{-S T} \in X_{N \backslash(S \cup T)}} u_{T}\left(x_{S}, y_{T}, x_{-S T}\right) \\
=\min _{x_{-} \in N \backslash S} u_{S}\left(y_{S}, x_{-S}\right)+\min _{x_{-T} \in N \backslash T} u_{T}\left(y_{T}, x_{-T}\right) .
\end{gathered}
$$

We can use this to derive that

$$
\begin{gathered}
w_{g}(S \cup T) \geq \max _{x_{S} \in X_{S}} \min _{x_{-S} \in N \backslash S} u_{S}\left(x_{S}, x_{-S}\right)+\max _{x_{T} \in X_{T}} \min _{x_{-T} \in N \backslash T} u_{T}\left(x_{T}, x_{-T}\right) \\
=w_{g}(S)+w_{g}(T) .
\end{gathered}
$$

This proves that $w_{g}$ is superadditive.

The two games $v_{g}$ and $w_{g}$ in example 3 are not only superadditive, but also have non-empty cores. The core of a coalitional game $(N, v)$ consists of divisions of the payoff $v(N)$ for the grand coalition in which each coalition $S \subset N$ of players gets at least their worth $v(S)$. In example 3 we found that $v_{g}(N)=w_{g}(N)=3$. If we divide this equally among the three players, 
giving them 1 each, then every single player gets at least his worth (which is $\frac{1}{2}$ in the game $v_{g}$ and 0 in the game $w_{g}$ ) and every 2-player coalition gets 2 , whereas its worth in $v_{g}$ equals 1 and that in $w_{g}$ equals 0 . Hence, this equal division is in the core of both games. It is not true in general, however, that the games $v_{g}$ and $w_{g}$ have non-empty cores. This is illustrated in the following example.

Example 4 Consider the following three-player strategic game g, in which player 1 is the row player, player 2 the column player, and player 3 chooses the matrix to the left or that to the right.

\begin{tabular}{|c|c|c|}
\hline$\alpha_{3}$ & $\alpha_{2}$ & $\beta_{2}$ \\
\hline$\alpha_{1}$ & $(1,1,0)$ & $(0,0,0)$ \\
\hline$\beta_{1}$ & $(1,0,1)$ & $(1,0,1)$ \\
\hline
\end{tabular}

\begin{tabular}{|c|c|c|}
\hline$\beta_{3}$ & $\alpha_{2}$ & $\beta_{2}$ \\
\hline$\alpha_{1}$ & $(1,1,0)$ & $(0,1,1)$ \\
\hline$\beta_{1}$ & $(0,0,0)$ & $(0,1,1)$ \\
\hline
\end{tabular}

Following the procedure as explained in detail in example 3, we derive that

$$
\begin{gathered}
v_{g}(i)=w_{g}(i)=0 \text { for each } i \in N, \\
v_{g}(i, j)=w_{g}(i, j)=2 \text { for each pair } i, j \in N, \\
\text { and } v_{g}(N)=w_{g}(N)=2 .
\end{gathered}
$$

Note that in any core-division of the worth $v_{g}(N)=w_{g}(N)=2$, each individual player would have to get no less than 0 and any two players should get at least 2 together. It is clearly impossible to meet all these conditions simultaneously. Hence, the cores of the games $\left(N, v_{g}\right)$ and $\left(N, w_{g}\right)$ are empty.

We continue this section by characterizing the two methods to associate a coalitional game with every strategic game. We define a method in general as a map $\phi: G \rightarrow \Gamma$ that associates a coalitional game $\phi(g) \in \Gamma^{N}$ with every strategic game $g \in G^{N}$. We denote the von Neumann and Morgenstern (1944) method by $\Psi_{V}$ and our method by $\Psi_{\underline{V}}$. Hence, $\Psi_{V}(g)=v_{g}$ and $\Psi_{\underline{V}}(g)=w_{g}$ for all $g \in G^{N}$. To characterize these two methods, we use properties that are derived from the properties we used in section 2 to characterize the value function and the lower value function. 
B1 Individual objectivity. For every $g \in G^{N}$, if a player $i \in N$ is such that $u_{i}(x)=c$, for all $x \in X_{N}$, then $\phi(g)(i)=c$.

B2 Monotonicity. If $g=\left(N,\left(X_{i}\right)_{i \in N},\left(u_{i}\right)_{i \in N}\right) \in G^{N}$ and

$$
g^{\prime}=\left(N,\left(X_{i}\right)_{i \in N},\left(u_{i}^{\prime}\right)_{i \in N}\right) \in G^{N}
$$

are two strategic games such that $u_{i} \geq u_{i}^{\prime}$ for some player $i \in N$, then for this player $i$ it holds that $\phi(g)(i) \geq \phi\left(g^{\prime}\right)(i)$.

B3 Irrelevance of dominated strategies. In a game $g \in G^{N}$, a strategy $x_{i} \in X_{i}$ of player $i$ is dominated if there exists a convex combination $y$ of the other strategies of player $i$, with the property that $u_{i}\left(x_{i}, x_{N \backslash i}\right) \leq u_{i}\left(y, x_{N \backslash i}\right)^{9}$ for all $x_{N \backslash i} \in X_{N \backslash i}$. For any $g \in G^{N}$ and player $i \in N$, if strategy $x_{i} \in X_{i}$ is dominated, then $\phi(g)(i)=\phi\left(g^{\prime}\right)(i)$, where $g^{\prime} \in G^{N}$ is the game that is obtained from $g$ by deleting strategy $x_{i}$.

B4 Irrelevance of strongly dominated strategies. In a game $g \in G^{N}$, a strategy $x_{i} \in X_{i}$ of player $i$ is strongly dominated if there exists a strategy $x_{i}^{\prime} \in X_{i}, x_{i}^{\prime} \neq x_{i}$, such that $u_{i}\left(x_{i}^{\prime}, x_{N \backslash i}\right) \geq u_{i}\left(x_{i}, x_{N \backslash i}\right)$ for all $x_{N \backslash i} \in X_{N \backslash i}$. For any $g \in G^{N}$ and player $i \in N$, if strategy $x_{i} \in X_{i}$ is strongly dominated, then $\phi(g)(i)=\phi\left(g^{\prime}\right)(i)$, where $g^{\prime} \in G^{N}$ is the game that is obtained from $g$ by deleting strategy $x_{i}$.

B5 Irrelevance of dominated threats. In a game $g \in G^{N}$, a strategy $x_{j} \in X_{j}$ of a player $j$ is a dominated threat to player $i \neq j$ if there exists a convex combination $y$ of the other strategies of player $j$, with the property that $u_{i}\left(y, x_{N \backslash j}\right) \leq u_{i}\left(x_{j}, x_{N \backslash j}\right)$ for all $x_{N \backslash j} \in X_{N \backslash j}$. For any $g \in G^{N}$ and players $i, j \in N, i \neq j$, if strategy $x_{j} \in X_{j}$ is a dominated threat to player $i$, then $\phi(g)(i)=\phi\left(g^{\prime}\right)(i)$, where $g^{\prime} \in G^{N}$ is the game that is obtained from $g$ by deleting strategy $x_{j}$.

\footnotetext{
${ }^{9} u_{i}\left(y, x_{N \backslash i}\right):=\sum_{\hat{x}_{i} \in X_{i}} y\left(\hat{x}_{i}\right) u_{i}\left(\hat{x}_{i}, x_{N \backslash i}\right)$, where $y=\sum_{\hat{x}_{i} \in X_{i}} y\left(\hat{x}_{i}\right) \hat{x}_{i}$. Note that $y\left(x_{i}\right)=0, y\left(\hat{x}_{i}\right) \geq 0$, for all $\hat{x}_{i} \in X_{i}$, and $\sum_{\hat{x}_{i} \in X_{i}} y\left(\hat{x}_{i}\right)=1$. Observe that $y$ is simply a mixed strategy of player $i$.
} 
B6 Irrelevance of weakly dominated threats. In a game $g \in G^{N}$, a strategy $x_{j} \in X_{j}$ of a player $j$ is a weakly dominated threat to player $i \neq j$ if for every $x_{N \backslash j} \in X_{N \backslash j}$ there exists a strategy $x_{j}^{\prime} \in X_{j}, x_{j}^{\prime} \neq x_{j}$, such that $u_{i}\left(x_{j}^{\prime}, x_{N \backslash j}\right) \leq u_{i}\left(x_{j}, x_{N \backslash j}\right)$. For any $g \in G^{N}$ and players $i, j \in N, i \neq j$, if strategy $x_{j} \in X_{j}$ is a weakly dominated threat to player $i$, then $\phi(g)(i)=\phi\left(g^{\prime}\right)(i)$, where $g^{\prime} \in G^{N}$ is the game that is obtained from $g$ by deleting strategy $x_{j}$.

Individual objectivity states that if player $i$ gets the same payoff for any possible strategy tuple, then in the associated coalitional game the worth of the coalition consisting of player $i$ only is equal to this amount. Monotonicity states that the worth of player $i$ in the associated coalitional game does not decrease if his payoff in the strategic game weakly increases for all possible strategy tuples. Irrelevance of dominated strategies and irrelevance of strongly dominated strategies mean that a player's worth in the coalitional game does not change if in the strategic game he loses the ability to use a strategy that was weakly worse for him than another one of his (mixed) strategies. Irrelevance of dominated strategies and irrelevance of strongly dominated strategies are derived from row dominance (A3) and weak row dominance (A8), respectively. Correspondingly, irrelevance of dominated strategies implies irrelevance of strongly dominated strategies but not the other way around. Irrelevance of dominated threats and irrelevance of weakly dominated threats are derived from column dominance (A5) and strong column dominance (A9), but they are adapted to be used in games with more than two players. They state that a player $i$ 's worth in the associated coalitional game is not affected if another player $j$ is prohibited from using a strategy whose deletion does not change player $i$ 's worst-case scenario. Irrelevance of weakly dominated threats is the stronger property of the two, as every threat that is dominated is also weakly dominated. Both irrelevance of dominated strategies and irrelevance of dominated threats make sense only in an environment where it is reasonable to assume that players can use mixed strategies, whereas irrelevance of strongly dominated strategies and irrelevance of weakly dominated threats are more adequate 
in situations in which players can only use pure strategies. To understand the relevance of properties B3 through B6, note that the worth of a player in the game $\phi(g)$ is interpreted as the payoff that this player can guarantee himself.

We need one additional property that has no equivalent in our characterizations of the (lower) value. It appears because we have to consider coalitions consisting of more than one player in the setting of the current section. We need some additional notation to introduce this property. Let $g=\left(N,\left\{X_{i}\right\}_{i \in N},\left\{u_{i}\right\}_{i \in N}\right) \in G^{N}$ and $S \subset N, S \neq \emptyset$. To study the opportunities of the members of $S$ as a group, we introduce a new player $p(S)$ with strategy set $X_{p(S)}:=X_{S}$ and utility function $u_{p(S)}: \prod_{j \in(N \backslash S) \cup\{p(S)\}} X_{j} \rightarrow \mathbb{R}$ defined by $u_{p(S)}\left(x_{N \backslash S}, x_{p(S)}\right)=u_{S}\left(x_{N \backslash S}, x_{S}\right)$ for all $x_{N \backslash S} \in X_{N \backslash S}$ and all $x_{p(S)}=x_{S} \in X_{S}=X_{p(S)}$. Denote $N(S):=(N \backslash S) \cup\{p(S)\}$. The game $g(S) \in G^{N(S)}$ is defined by $g(S)=\left(N(S),\left\{X_{i}\right\}_{i \in N(S)},\left\{u_{i}\right\}_{i \in N(S)}\right)$. The property merge invariance states that the worth of coalition $S$ in the original strategic game $g$ is the same as that of player $p(S)$ in the game $g(S)$. Its interpretation is that a coalition of players cannot influence its worth by merging and acting as one player. Its validity derives from the very interpretation of a coalition in a coalitional game as a group of players acting in the best interests of the group.

B7 Merge invariance. Let $g=\left(N,\left\{X_{i}\right\}_{i \in N},\left\{u_{i}\right\}_{i \in N}\right) \in G^{N}$ and $S \subset N$, $S \neq \emptyset$. Then $\phi(g)(S)=\phi(g(S))(p(S))$, where $g(S)$ is the strategic game that is obtained from $g$ by considering the coalition $S$ as a single player.

The properties introduced above can be used to axiomatically characterize the two methods $\Psi_{V}$ and $\Psi_{V}$. We only provide a proof of one of the following theorems, as their structure is similar to the proofs of Theorems 2 and 4 , respectively, and providing one proof suffices to illustrate the role of the extra property merge invariance.

Theorem 6 The method $\Psi_{V}$ is the unique method satisfying individual objectivity (B1), monotonicity (B2), irrelevance of dominated strategies (B3), 
irrelevance of dominated threats (B5), and merge invariance (B7).

Theorem 7 The method $\Psi_{V}$ is the unique method satisfying individual objectivity (B1), monotonicity (B2), irrelevance of strongly dominated strategies (B4), irrelevance of weakly dominated threats (B6), and merge invariance $(B 7)$.

Proof. First, we show that $\Psi_{\underline{V}}$ satisfies the five properties. Let $g \in G^{N}$ and $i \in N$ be such that $u_{i}(x)=c$, for all $x \in X_{N}$. Then, in the matrix $A_{i}$ of the game $g_{i}$, all entries are equal to $c$. The lower value of this matrix equals $c$. Hence, $\Psi_{\underline{V}}(g)(i)=w_{g}(i)=c$, which shows that $\Psi_{\underline{V}}$ satisfies B1.

Now, let $g=\left(N,\left(X_{i}\right)_{i \in N},\left(u_{i}\right)_{i \in N}\right) \in G^{N}$ and $g^{\prime}=\left(N,\left(X_{i}\right)_{i \in N},\left(u_{i}^{\prime}\right)_{i \in N}\right) \in$ $G^{N}$ be two strategic games such that $u_{i} \geq u_{i}^{\prime}$ for player $i \in N$. Then, $A_{i} \geq A_{i}^{\prime}$, where $A_{i}$ denotes the matrix of the game $g_{i}$ and $A_{i}^{\prime}$ denotes the matrix of the game $g_{i}^{\prime}$. It now follows from monotonicity of the lower value function that $\Psi_{\underline{V}}(g)(i)=w_{g}(i) \geq w_{g^{\prime}}(i)=\Psi_{\underline{V}}\left(g^{\prime}\right)(i)$. This proves that $\Psi_{\underline{V}}$ satisfies B2.

To see that $\Psi_{\underline{V}}$ satisfies B4, note that if strategy $x_{i}$ for player $i$ is strongly dominated in the game $g \in G^{N}$, then it corresponds to a strongly dominated row in the matrix $A_{i}$ of the game $g_{i}$. Hence, by weak row dominance of the lower value function, it holds that $\Psi_{\underline{V}}(g)(i)=w_{g}(i)=w_{g^{\prime}}(i)=\Psi_{\underline{V}}\left(g^{\prime}\right)(i)$, where $g^{\prime} \in G^{N}$ is the game that is obtained from $g$ by deleting strategy $x_{i}$.

To see that $\Psi_{\underline{V}}$ satisfies B6, note that if strategy $x_{j} \in X_{j}$ of a player $j$ is a weakly dominated threat to player $i \neq j$ in the game $g \in G^{N}$, then for all $x_{N \backslash i, j} \in X_{N \backslash i, j}$ strategy $\left(x_{j}, x_{N \backslash i, j}\right)$ corresponds to a weakly dominated column in the matrix $A_{i}$ of the game $g_{i}$. Hence, using strong column dominance of the lower value function (repeatedly), we can eliminate the strategy (column) $\left(x_{j}, x_{N \backslash i, j}\right)$ for each $x_{N \backslash i, j} \in X_{N \backslash i, j}$, without changing the lower value. The matrix that we have left is that corresponding to the game $g^{\prime} \in G^{N}$ that is obtained from $g$ by deleting strategy $x_{j}$. For this game, we then have $\Psi_{\underline{V}}(g)(i)=w_{g}(i)=w_{g^{\prime}}(i)=\Psi_{\underline{V}}\left(g^{\prime}\right)(i)$.

It follows easily that $\Psi_{\underline{V}}$ satisfies B7 by noting that the matrix $A_{S}$ of the strategic game $g_{S}$ derived from $g$ and the matrix $A_{p(S)}$ of the strategic 
game $g(S)_{p(S)}$ derived from $g(S)$ are, in fact, the same.

We now proceed to show that any method satisfying the five properties must coincide with $\Psi_{\underline{V}}$. Let $\phi: G \rightarrow \Gamma$ be a method satisfying individual objectivity, monotonicity, irrelevance of strongly dominated strategies, irrelevance of weakly dominated threats, and merge invariance. Take a finite strategic game $g=\left(N,\left(X_{i}\right)_{i \in N},\left(u_{i}\right)_{i \in N}\right) \in G^{N}$ and fix a nonempty coalition $S \subset N$. If $S=N$, then merge invariance, irrelevance of strongly dominated strategies, and individual objectivity clearly imply that $\phi(g)(N)=w_{g}(N)=\Psi_{\underline{V}}(g)(N)$. Assume now that $S \neq N$. We will prove that $\phi(g)(S)=w_{g}(S)=\Psi_{\underline{V}}(g)(S)$ has to hold.

Consider the game $g(S)=\left(N(S),\left\{X_{i}\right\}_{i \in N(S)},\left\{u_{i}\right\}_{i \in N(S)}\right)$ that is obtained from $g$ by considering the coalition $S$ as a single player $p(S)$. Because $\phi$ satisfies merge invariance, we know that $\phi(g)(S)=\phi(g(S))(p(S))$.

We know from the definition of $g(S)$ that the matrix $A_{S}$ of the strategic game $g_{S}$ derived from $g$ and the matrix $A_{p(S)}$ of the strategic game $g(S)_{p(S)}$ derived from $g(S)$ are the same. From this we can conclude that $w_{g}(S)=$ $\underline{V}\left(A_{S}\right)=\underline{V}\left(A_{p(S)}\right)=w_{g(S)}(p(S))$. Now, let $\bar{x}=\left(\bar{x}_{i}\right)_{i \in N} \in \prod_{i \in N} X_{i}$ be a strategy such that the lower value of $A_{S}$ is obtained in the row corresponding to strategy $\bar{x}_{S}$ for coalition $S$ and the column corresponding to strategy $\bar{x}_{N \backslash S}$ for coalition $N \backslash S$. Then the lower value of $A_{p(S)}$ is obtained in the row corresponding to strategy $\bar{x}_{p(S)}=\bar{x}_{S} \in X_{p(S)}$ for player $p(S)$ and the column corresponding to strategy $\bar{x}_{N \backslash S}$ for coalition $N \backslash S$.

Let $g_{1}$ be the game that is obtained from the game $g(S)$ by bounding the utility of player $p(S)$ from above by $w_{g}(S)$, i.e.,

$$
g_{1}=\left(N(S),\left\{X_{i}\right\}_{i \in N(S)},\left\{u_{i}\right\}_{i \in N \backslash S}, u_{p(S)}^{\prime}\right),
$$

where $N(S)=(N \backslash S) \cup\{p(S)\}$ and

$$
u_{p(S)}^{\prime}\left(x_{N \backslash S}, x_{p(S)}\right)=\min \left\{u_{S}\left(x_{N \backslash S}, x_{S}\right), w_{g}(S)\right\}
$$

for all $x_{N \backslash S} \in X_{N \backslash S}$ and all $x_{p(S)}=x_{S} \in X_{S}=X_{p(S)}$. Because $\phi$ satisfies monotonicity, we know that $\phi(g(S))(p(S)) \geq \phi\left(g_{1}\right)(p(S))$.

Now, note that $w_{g}(S)=\max _{x_{p(S)} \in X_{p(S)}} \min _{x_{N \backslash S} \in X_{N \backslash S}} u_{p(S)}\left(x_{p(S)}, x_{N \backslash S}\right)$ is obtained at $\left(\bar{x}_{p(S)}, \bar{x}_{N \backslash S}\right)$, so that $\min _{x_{N \backslash S} \in X_{N \backslash S}} u_{p(S)}\left(\bar{x}_{p(S)}, x_{N \backslash S}\right)=w_{g}(S)$ 
and $u_{p(S)}\left(\bar{x}_{p(S)}, x_{N \backslash S}\right) \geq w_{g}(S)$ for all $x_{N \backslash S} \in X_{N \backslash S}$. Hence,

$$
u_{p(S)}^{\prime}\left(\bar{x}_{p(S)}, x_{N \backslash S}\right)=w_{g}(S)
$$

for all $x_{N \backslash S} \in X_{N \backslash S}$. Moreover, we have $u_{p(S)}^{\prime}\left(x_{p(S)}, x_{N \backslash S}\right) \leq w_{g}(S)$ for all $x_{N \backslash S} \in X_{N \backslash S}$ and all $x_{p(S)} \in X_{p(S)}$. Hence, every strategy $x_{p(S)} \in X_{p(S)}$, $x_{p(S)} \neq \bar{x}_{p(S)}$, is strongly dominated by strategy $\bar{x}_{p(S)}$. Because $\phi$ satisfies irrelevance of strongly dominated strategies, we can eliminate all the strongly dominated strategies of player $p(S)$ without changing the worth of $p(S)$ in the image of the game under $\phi$. Hence, $\phi\left(g_{1}\right)(p(S))=\phi\left(g_{2}\right)(p(S))$, where $g_{2}$ is the game that is obtained from $g_{1}$ by deleting all strategies of player $p(S)$ except strategy $\bar{x}_{p(S)}$.

Now, in the game $g_{2}$, for every player $j \neq p(S)$ it holds that every strategy $x_{j} \in X_{j} \backslash \bar{x}_{j}$ is a weakly dominated threat to player $p(S)$, because $u_{p(S)}^{\prime}\left(\bar{x}_{p(S)}, \bar{x}_{N \backslash S}\right)=\min _{x_{N \backslash S} \in X_{N \backslash S}} u_{p(S)}^{\prime}\left(\bar{x}_{p(S)}, x_{N \backslash S}\right) .{ }^{10}$ Because $\phi$ satisfies irrelevance of weakly dominated threats, we can eliminate all the weakly dominated threats to player $p(S)$ without changing the worth of $p(S)$ in the image of the game under $\phi$. Hence, $\phi\left(g_{2}\right)(p(S))=\phi\left(g_{3}\right)(p(S))$, where $g_{3}$ is the game that is obtained from $g_{2}$ by deleting all strategies $x_{j} \in X_{j} \backslash \bar{x}_{j}$ for every player $j \in N \backslash S$.

In the game $g_{3}$ every player $j$ has exactly one strategy, $\bar{x}_{j}$. Hence, for this game we can use individual objectivity of $\phi$ to derive that

$$
\phi\left(g_{3}\right)(p(S))=u_{p(S)}^{\prime}(\bar{x}) .
$$

Putting everything together, we see that we proved that $\phi(g)(S)=$ $\phi(g(S))(p(S)) \geq \phi\left(g_{1}\right)(p(S))=\phi\left(g_{2}\right)(p(S))=\phi\left(g_{3}\right)(p(S))=u_{p(S)}^{\prime}(\bar{x})=$ $w_{g}(S)=\Psi_{\underline{V}}(g)(S)$.

To finish the proof of the Theorem, we need to prove that $\phi(g)(S) \leq$ $\Psi_{\underline{V}}(g)(S)$.

Consider again the game $g(S)=\left(N(S),\left\{X_{i}\right\}_{i \in N(S)},\left\{u_{i}\right\}_{i \in N(S)}\right)$ that is obtained from $g$ by considering the coalition $S$ as a single player $p(S)$. We have already seen that $\phi(g)(S)=\phi(g(S))(p(S))$ and $w_{g}(S)=w_{g(S)}(p(S))$.

\footnotetext{
${ }^{10}$ In fact, $u_{p(S)}^{\prime}\left(\bar{x}_{p(S)}, \bar{x}_{N \backslash S}\right)=u_{p(S)}^{\prime}\left(\bar{x}_{p(S)}, x_{N \backslash S}\right)=w_{g}(S)$ for all $x_{N \backslash S} \in X_{N \backslash S}$.
} 
We will define a new game $g_{4}$ by adding a strategy $x_{i}^{*} \notin X_{i}$ for each player $i \in N \backslash S$. The strategies $x_{i}^{*}$ are introduced as additional threats to player $p(S)$. We add these strategies one by one. Without loss of generality, we assume that $N \backslash S=\{1,2, \ldots, k\}$, where $k$ denotes the number of players in $N \backslash S$.

We first define the game $g_{1}^{*}$, by adding strategy $x_{1}^{*}$ for player 1 . The payoffs to player $p(S)$ in the game $g_{1}^{*}$ are as in the game $g(S)$ when player 1 plays a strategy $x_{1} \in X_{1}$. When player 1 plays his strategy $x_{1}^{*}$, then the payoffs to player $p(S)$ are defined by

$$
u_{p(S)}^{1}\left(x_{p(S)}, x_{1}^{*},\left(x_{i}\right)_{i \in\{2,3, \ldots, k\}}\right)=\min _{x_{1} \in X_{1}}\left\{u_{p(S)}\left(x_{p(S)}, x_{1},\left(x_{i}\right)_{i \in\{2,3, \ldots, k\}}\right)\right\},
$$

where $x_{i} \in X_{i}$ for all $i \in\{2,3, \ldots, k\}$ and $x_{p(S)} \in X_{p(S)}$. In the game $g_{1}^{*}$, it holds that strategy $x_{1}^{*}$ is a weakly dominated threat to player $p(S)$. Because $\phi$ satisfies irrelevance of weakly dominated threats, we can eliminate this weakly dominated threat to player $p(S)$ from $g_{1}^{*}$ without changing the worth of $p(S)$ in the image of the game under $\phi$. This shows that $\phi\left(g_{1}^{*}\right)(p(S))=$ $\phi(g(S))(p(S))$.

Now, let $2 \leq j \leq k$ and suppose that we have added a strategy $x_{i}^{*}$ for each player $i=1,2, \ldots j-1$ and defined the corresponding games $g_{i}^{*}$ with payoff functions $u_{p(S)}^{i}$ for player $p(S)$ so that in each game $g_{i}^{*}$ strategy $x_{i}^{*}$ is a weakly dominated threat to player $p(S)$ and $\phi\left(g_{i}^{*}\right)(p(S))=\phi(g(S))(p(S))$. To obtain the game $g_{j}^{*}$, we add a strategy $x_{j}^{*}$ for player $j$ and define the payoffs to player $p(S)$ to be as in the game $g_{j-1}^{*}$ when player $j$ plays a strategy $x_{j} \in X_{j}$, and when player $j$ plays his strategy $x_{j}^{*}$ they are

$$
\begin{gathered}
u_{p(S)}^{j}\left(x_{p(S)},\left(y_{i}\right)_{i \in\{1, \ldots, j-1\}}, x_{j}^{*},\left(x_{i}\right)_{i \in\{j+1, \ldots, k\}}\right)= \\
\min _{x_{j} \in X_{j}}\left\{u_{p(S)}^{j-1}\left(x_{p(S)},\left(y_{i}\right)_{i \in\{1, \ldots, j-1\}}, x_{j},\left(x_{i}\right)_{i \in\{j+1, \ldots, k\}}\right)\right\},
\end{gathered}
$$

where $y_{i} \in X_{i} \cup\left\{x_{i}^{*}\right\}$ for all $i \in\{1, \ldots, j-1\}, x_{i} \in X_{i}$ for all $i \in\{j+1, \ldots, k\}$, and $x_{p(S)} \in X_{p(S)}$. In the game $g_{j}^{*}$, it holds that strategy $x_{j}^{*}$ is a weakly dominated threat to player $p(S)$. Because $\phi$ satisfies irrelevance of weakly dominated threats, we can eliminate this weakly dominated threat to player 
$p(S)$ from $g_{j}^{*}$ without changing the worth of $p(S)$ in the image of the game under $\phi$. This shows that $\phi\left(g_{j}^{*}\right)(p(S))=\phi\left(g_{j-1}^{*}\right)(p(S))=\phi(g(S))(p(S))$.

The game $g_{4}$ is the game $g_{k}^{*}$ which emerges from the procedure described above after a strategy $x_{i}^{*}$ has been added for each player $i \in N \backslash S$. The payoffs to player $p(S)$ in the game $g_{4}$ are denoted by $u_{p(S)}^{\prime}:=u_{p(S)}^{k}$. We have that $\phi\left(g_{4}\right)(p(S))=\phi(g(S))(p(S))$. Also, it holds that

$$
\begin{gathered}
u_{p(S)}^{\prime}\left(x_{p(S)},\left(x_{i}^{*}\right)_{i \in N \backslash S}\right)= \\
u_{p(S)}^{k}\left(x_{p(S)},\left(x_{i}^{*}\right)_{i \in N \backslash S}\right)=\min _{x_{k} \in X_{k}}\left\{u_{p(S)}^{k-1}\left(x_{p(S)},\left(x_{i}^{*}\right)_{i \in\{1, \ldots, k-1\}}, x_{k}\right)\right\}= \\
\min _{x_{k} \in X_{k} x_{k-1} \in X_{k-1}}\left\{u_{p(S)}^{k-2}\left(x_{p(S)},\left(x_{i}^{*}\right)_{i \in\{1, \ldots, k-2\}}, x_{k-1}, x_{k}\right)\right\}= \\
\ldots=\min _{x_{N \backslash S} \in X_{N \backslash S}} u_{p(S)}\left(x_{p(S)}, x_{N \backslash S}\right) \leq w_{g(S)}(p(S))
\end{gathered}
$$

for all $x_{p(S)} \in X_{p(S)}$. We will use this later.

Let $g_{5}$ be the game that is obtained from the game $g_{4}$ by bounding the utility of player $p(S)$ from below by $w_{g}(S)$, i.e., the payoff function of player $p(S)$ is $u_{p(S)}^{\prime \prime}\left(x_{p(S)}, y_{N \backslash S}\right)=\max \left\{u_{p(S)}^{\prime}\left(x_{p(S)}, y_{N \backslash S}\right), w_{g}(S)\right\}$ for all $x_{p(S)} \in$ $X_{p(S)}$ and all $y_{N \backslash S} \in \prod_{i \in N \backslash S} X_{i} \cup\left\{x_{i}^{*}\right\}$. Because $\phi$ satisfies monotonicity, we know that $\phi\left(g_{5}\right)(p(S)) \geq \phi\left(g_{4}\right)(p(S))$.

Now, note that the game $g_{5}$ has been constructed in such a way that $u_{p(S)}^{\prime \prime}\left(x_{p(S)}, x_{i}^{*}, y_{N \backslash(S \cup i)}\right)=\min _{x_{i} \in X_{i}} u_{p(S)}^{\prime \prime}\left(x_{p(S)}, x_{i}, y_{N \backslash(S \cup i)}\right)$ for all $i \in N \backslash S$, all $x_{p(S)} \in X_{p(S)}$, and all $y_{N \backslash(S \cup i)} \in \prod_{j \in N \backslash(S \cup i)} X_{j} \cup\left\{x_{j}^{*}\right\}$. Hence, every strategy $x_{i} \in X_{i}$ is a weakly dominated threat to player $p(S)$ for every player $i \neq p(S)$. Because $\phi$ satisfies irrelevance of weakly dominated threats, we can eliminate all the weakly dominated threats to player $p(S)$ without changing the worth of $p(S)$ in the image of the game under $\phi$. Hence, $\phi\left(g_{5}\right)(p(S))=\phi\left(g_{6}\right)(p(S))$, where $g_{6}$ is the game that is obtained from $g_{5}$ by deleting all strategies $x_{i} \in X_{i}$ for every player $i \in N \backslash S$.

In the game $g_{6}$ all players $i \neq p(S)$ have only one strategy, strategy $x_{i}^{*}$, and as $u_{p(S)}^{\prime}\left(x_{p(S)},\left(x_{i}^{*}\right)_{i \in N \backslash S}\right) \leq w_{g(S)}(p(S))=w_{g}(S)$, it holds that

$$
u_{p(S)}^{\prime \prime}\left(x_{p(S)},\left(x_{i}^{*}\right)_{i \in N \backslash S}\right)=\max \left\{u_{p(S)}^{\prime}\left(x_{p(S)},\left(x_{i}^{*}\right)_{i \in N \backslash S}\right), w_{g}(S)\right\}=w_{g}(S)
$$


for every $x_{p(S)} \in X_{p(S)}$. Hence, the conditions for individual objectivity are satisfied and we can conclude that $\phi\left(g_{6}\right)(p(S))=w_{g}(S)$.

Putting everything together, we see that we proved that $\phi(g)(S)=$ $\phi(g(S))(p(S))=\phi\left(g_{4}\right)(p(S)) \leq \phi\left(g_{5}\right)(p(S))=\phi\left(g_{6}\right)(p(S))=w_{g}(S)=$ $\Psi_{\underline{V}}(g)(S)$.

This finishes the proof.

We already pointed out that irrelevance of dominated strategies implies irrelevance of strongly dominated strategies and that irrelevance of weakly dominated threats implies irrelevance of dominated threats. Hence, Theorems 6 and 7 allow us to conclude that $\Psi_{V}$ does not satisfy irrelevance of weakly dominated threats and that $\Psi_{\underline{V}}$ does not satisfy irrelevance of dominated strategies.

Analogously to our characterizations in section 2, we can replace the monotonicity property in Theorems 6 and 7 by the two following properties.

B8 Elimination of own strategies. For every strategic game $g \in G^{N}$, every player $i \in N$, and every strategy $x_{i} \in X_{i}$, it holds that $\phi(g)(i) \geq$ $\phi\left(g^{\prime}\right)(i)$, where $g^{\prime} \in G^{N}$ is that game that is obtained from $g$ by deleting strategy $x_{i}$.

B9 Elimination of others' strategies. For every strategic game $g \in$ $G^{N}$, any two players $i, j \in N, i \neq j$, and every strategy $x_{j} \in X_{j}$, it holds that $\phi(g)(i) \leq \phi\left(g^{\prime}\right)(i)$, where $g^{\prime} \in G^{N}$ is that game that is obtained from $g$ by deleting strategy $x_{j}$.

\section{References}

Aumann RJ (1961) The core of a cooperative game without side payments. In: Tucker AW, Luce RD (eds.) Contributions to the Theory of Games IV. Princeton University Press, pp. 287-324

Aumann RJ (1967) A survey of cooperative games without side payments. In: Shubik M (ed.) Essays in Mathematical Economics. Princeton University Press, pp. 3-27 
Bergantiños G, García-Jurado I (1995) Estudio comparativo de diversas funciones características asociadas a un juego en forma normal. Investigaciones Económicas 19:127-138

Borm P, Tijs SH (1992) Strategic claim games corresponding to an NTUgame. Games and Economic Behavior 4:58-71

Harsanyi JC (1963) A simplified bargaining model for the n-person cooperative game. International Economic Review 4:194-220

Myerson RB (1978) Threat equilibria and fair settlements cooperative games. Mathematics of Operations Research 3:265-274

Myerson RB (1991) Game theory, analysis of conflict. Harvard University Press

Tijs SH (1981) A characterization of the value of zero-sum two-person games. Naval Research Logistics Quarterly 28:153-156

van Damme E, Furth D (2002) Game theory and the market. In: Borm P, Peters H (eds.) Chapters in Game Theory. Kluwer Academic Publishers, pp. $51-81$

Vilkas EI (1963) Axiomatic definition of the value of a matrix game. Theory of Probability and its Applications 8:304-307

von Neumann J (1928) Zur theorie der gesellschaftsspiele. Mathematische Annalen 100:295-320

von Neumann J, Morgenstern O (1944) Theory of games and economic behavior. Princeton University Press 